\title{
Respiratory Control and the Onset of Periodic Breathing
}

\author{
A. C. Fowler* \\ MACSI, University of Limerick, Limerick, Ireland \\ OCIAM, Mathematical Institute, University of Oxford, Oxford, UK
}

\begin{abstract}
We analyse a reduced version of the Grodins et al. control model [14] of respiration involving only $\mathrm{CO}_{2}$, and show that it can be dramatically simplified by the use of judicious approximations. In particular, we show that the conceptual basis of the popular model of Mackey and Glass [20] is at odds with the important transport processes of the Grodins model. Despite this, a realistic approximation of the Grodins model yields a Mackey-Glass type model with almost the same criterion for the onset of Cheyne-Stokes breathing.

While the reduced Grodins model does apparently provide a realistic mechanism for instability, consideration of the buffering effect of the blood-brain barrier appears to make it unlikely. We conclude that a realistic physiological model of Grodins type to explain Cheyne-Stokes breathing is not yet in place, and raise the question whether the bicarbonate buffering system has a rôle to play.
\end{abstract}

Keywords and phrases: periodic breathing, respiratory control, Cheyne-Stokes respiration, delay equation

Mathematics Subject Classification: 92C50

\section{Introduction}

Cheyne-Stokes breathing ([18]) is an oscillatory form of breathing in which a waxing and waning ventilation occurs for about 30 seconds, and this is followed by apnea (absence of breathing) for a further 30 seconds. This cycle then repeats periodically. It was first described by Cheyne [6] and Stokes [24] in patients with heart failure, and it also occurs in patients with stroke, and in climbers at high altitude (West et al. [27], [25]. It is generally accepted that increased circulatory delay and increased controller gain are both causes of the instability which provides the explanation of Cheyne-Stokes breathing, and a very simple model which represents this explicitly is that by Mackey and Glass [20]. The simplicity of this model, and the elegance of the simple stability criterion which results, has led to the Mackey-Glass model becoming a popular pedagogical tool (e.g., [23]); the purpose of the present paper is to illuminate the conceptual basis for this model, and suggest that this basis is less well founded than it might appear.

Many authors have modelled the control of respiration by the action of oxygen and carbon dioxide on peripheral and central chemoreceptors. The most physiologically complicated of these models is that of

\footnotetext{
*Corresponding author. E-mail: andrew.fowler@ul.ie
} 
Grodins et al. [14], which will provide a focus for our study, and to which we will refer as the Grodins model. Simpler models which all follow the same basic compartmental framework as the Grodins model have been developed by, for example, Mackey and Glass [20], Khoo et al. [17], Carley and Shannon [5], Longobardo et al. [19], ElHefnawy et al. [8], Batzel and Tran [1],[2],[3], and Dong and Langford [7]. Bental and Smith [4] have developed a model which includes an explicit description of the neural ventilatory controller.

In previous work (e.g., [9],[10],[11]), we have shown how the Grodins model can be simplified using asymptotic approximations based on a realistic assessment of the appropriate parameter values. Our purpose in the present paper is to show how the Mackey Glass model could also be derived from a more complex model, but that in fact such a derivation is inappropriate in humans.

The structure of the paper is as follows. In section 2 we describe a simplified form of the Grodins model, and we derive a dimensionless form of it, suitable for further approximation. This supersedes our earlier, more opaque, efforts in this direction [9]. In section 3, we describe the Mackey-Glass model, and we show how it can be derived from the Grodins model by suitable approximations. However, we show in section 4 that these approximations are inappropriate, but that a correct simplification nevertheless leads to a Mackey-Glass type model, and we give the analytic instability criterion for this case. In section 5 , we compare the different stability criteria, and in section 6 , we also suggest that instability by this route may not be physiologically relevant, and that the simplified versions of the model may yet be too oversimplified to be of clinical use.

\section{The Grodins model}

We begin by describing a simplified version of the Grodins model, similar in scope to those of Khoo et al. [17] and Batzel and Tran [1]. The full Grodins model was addressed by Fowler et al. [9], who showed in detail that such a simplification was possible. The model enunciated by Grodins et al. [14] describes the blood gas concentrations in four separate compartments, namely brain, (other) tissues, lungs and cerebro-spinal fluid: the scheme of the model is indicated in figure 1. Concentrations of $\mathrm{CO}_{2}$, $\mathrm{O}_{2}$ (and $\mathrm{N}_{2}$ ) are described by conservation laws which balance the transport between compartments via arteries or veins with the metabolic consumption or production rates (of $\mathrm{O}_{2}$ and $\mathrm{CO}_{2}$ respectively). In seeking to understand this model, we begin by focussing on the transport of $\mathrm{CO}_{2}$ only, and we also ignore complications associated with variable blood flow.

Three equations describing $\mathrm{CO}_{2}$ levels in the lung, brain and tissue compartments are Grodins et al.'s [14] equations (1.1), (1.4) and (1.7): when written in terms of partial pressures, these take the form

$$
\begin{aligned}
& K_{\mathrm{L}} \dot{P}_{\mathrm{aCO}_{2}}=-\dot{V} P_{\mathrm{aCO}_{2}}+863 K_{\mathrm{CO}_{2}} Q\left[P_{\mathrm{vCO}_{2}}-P_{\mathrm{aCO}_{2}}\right] \text {, } \\
& K_{\mathrm{CO}_{2}} K_{\mathrm{B}} \dot{P}_{\mathrm{BCO}_{2}}=M R_{\mathrm{BCO}_{2}}+K_{\mathrm{CO}_{2}} Q_{\mathrm{B}}\left[P_{\mathrm{aCO}_{2}}\left(t-\tau_{\mathrm{aB}}\right)-P_{\mathrm{BCO}_{2}}\right] \\
& -D_{\mathrm{CO}_{2}}\left[P_{\mathrm{BCO}_{2}}-P_{\mathrm{CSFCO}_{2}}\right] \\
& K_{\mathrm{CO}_{2}} K_{\mathrm{T}} \dot{P}_{\mathrm{TCO}_{2}}=M R_{\mathrm{TCO}_{2}}+\left(Q-Q_{B}\right) K_{\mathrm{CO}_{2}}\left[P_{\mathrm{aCO}_{2}}\left(t-\tau_{a T}\right)-P_{\mathrm{TCO}_{2}}\right] .
\end{aligned}
$$

They are supplemented by the flow conservation equation

$$
Q P_{\mathrm{vCO}_{2}}=Q_{\mathrm{B}} P_{\mathrm{BCO}_{2}}\left(t-\tau_{\mathrm{vB}}\right)+\left(Q-Q_{\mathrm{B}}\right) P_{\mathrm{TCO}_{2}}\left(t-\tau_{\mathrm{vT}}\right) .
$$

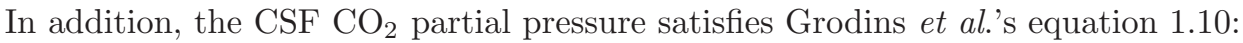

$$
K_{\mathrm{CSF}} k \alpha_{\mathrm{CO}_{2}} \dot{P}_{\mathrm{CSFCO}_{2}}=D_{\mathrm{CO}_{2}}\left(P_{\mathrm{BCO}_{2}}-P_{\mathrm{CSFCO}_{2}}\right),
$$

in which $K_{\mathrm{CSF}}$ is $\mathrm{CSF}$ volume, $k$ is a conversion factor from atmospheric pressure to $\mathrm{mm} \mathrm{Hg}$ (thus $k=1 / 760$ ), and $\alpha_{\mathrm{CO}_{2}}$ is a solubility coefficient for $\mathrm{CO}_{2}$ in CSF.

The variables $P$ represent partial pressures of the blood gas carbon dioxide, with the extra suffixes a, v, B, T and CSF denoting the various compartments: arterial, venous, brain, tissues and cerebro-spinal 


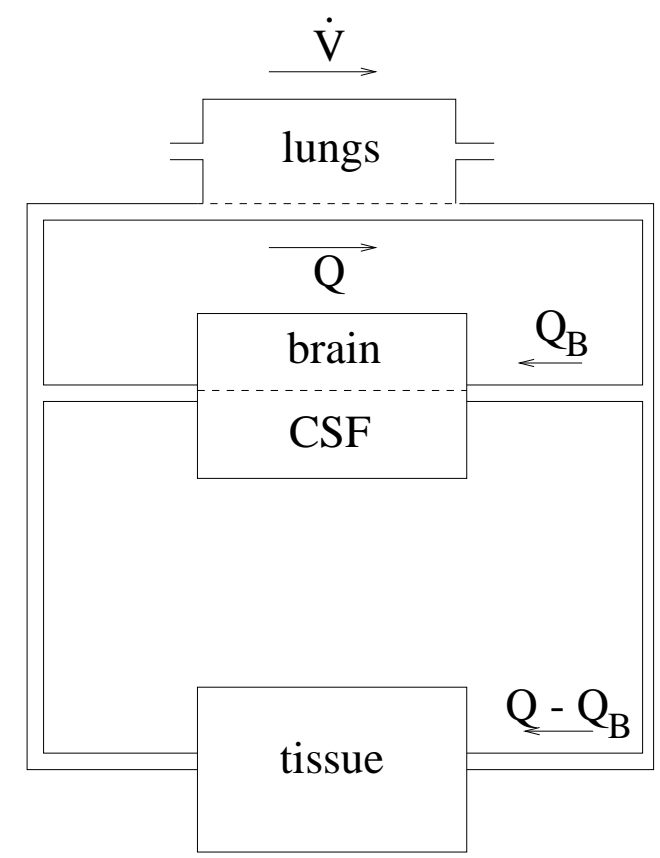

Figure 1. Compartment scheme of the Grodins model.

fluid. $\dot{V}$ represents the minute ventilation; $K_{L}, K_{B}, K_{T}$ and $K_{C S F}$ represent compartment volumes of lung, brain, tissues and cerebrospinal fluid respectively; $M R_{\mathrm{BCO}_{2}}$ and $M R_{\mathrm{TCO}_{2}}$ represent brain and tissue metabolic production rate of $\mathrm{CO}_{2} ; Q$ and $Q_{B}$ represent total blood flow and blood flow to the brain, respectively. The dissociation constant $K_{\mathrm{CO}_{2}}$ is a conversion factor from units of concentration (litres per litre) to units of partial pressure (conventionally measured as millimetres of mercury $(\mathrm{mm} \mathrm{Hg}$ ). The factor 863 arises in converting inhaled dry gas volumes at standard temperature and pressure (STPD) to body temperature and pressure saturated (BTPS) [26]. The diffusion constant $D_{\mathrm{CO}_{2}}$ measures the rate at which $\mathrm{CO}_{2}$ permeates through the blood brain barrier. The four delays $\tau_{\mathrm{aB}}, \tau_{a T}, \tau_{\mathrm{vB}}$ and $\tau_{\mathrm{vT}}$ represent the time taken for the blood to flow (arterially) from the heart and lungs to the brain and tissues, and to the lungs and heart from the brain and tissues, respectively. (The pulmonary circulation is not treated separately in the Grodins model.)

For variable blood flow, the delays are calculated as follows. Suppose that points $R$ and $S$ are joined by an artery of volume $V_{\mathrm{RS}}$ through which blood flows at a variable rate $Q(t)$. If the blood at point $S$ at time $t$ was at point $R$ at time $t-\tau_{\mathrm{RS}}$, then the volume $V$ of artery traversed satisfies $\frac{d V}{d s}=Q(s)$, $V=0$ at $s=t-\tau_{\mathrm{RS}}, V=V_{\mathrm{RS}}$ at $s=t$. Integrating this, we find $V_{\mathrm{RS}}=\int_{t-\tau_{\mathrm{RS}}}^{t} Q(s) d s$. If now a flow $Q(t)$ traverses a volume $V_{\mathrm{aB}}$ from the heart to the point $C$, and then branches as in figure 1 , so that a sub-flow $Q_{B}$ traverses a further arterial volume $\tilde{V}_{\mathrm{aB}}$ from $C$ to $B$, then the blood which is at $B$ at time $t$ is at $C$ at time $t-\tilde{\tau}_{\mathrm{aB}}$, and at the heart at time $t-\tau_{\mathrm{aB}}$, where

$$
V_{\mathrm{aB}}=\int_{t-\tau_{\mathrm{aB}}}^{t-\tilde{\tau}_{\mathrm{aB}}} Q(s) d s, \quad \tilde{V}_{\mathrm{aB}}=\int_{t-\tilde{\tau}_{\mathrm{aB}}}^{t} Q_{B}(s) d s,
$$

and these relations determine $\tau_{\mathrm{aB}}$. For steady blood flow, we simply have

$$
\tau_{\mathrm{aB}}=\frac{V_{\mathrm{aB}}}{Q}+\frac{\tilde{V}_{\mathrm{aB}}}{Q_{B}},
$$


and there are similar expressions for the other delays.

The ventilation $\dot{V}$ responds to blood gas concentrations in two ways. There are central chemoreceptors located in the medulla area of the brainstem, and peripheral chemoreceptors located in the arch of the carotid artery, in the neck. The peripheral chemoreceptors respond to both carbon dioxide and oxygen, and the two responses are coupled. Essentially, $\dot{V}$ is an increasing function of $\mathrm{CO}_{2}$ partial pressure at the carotid artery, and can be reasonably represented by the piecewise smooth function

$$
\dot{V}=G\left[P-P_{0}\right]_{+},
$$

where the gain $G$ is a decreasing function of oxygen concentration, as illustrated in figure 2. (2.6) is essentially the form of controller used by Batzel and Tran [1]. The forms used by Grodins et al. [14] were similar, but algebraically more complicated.

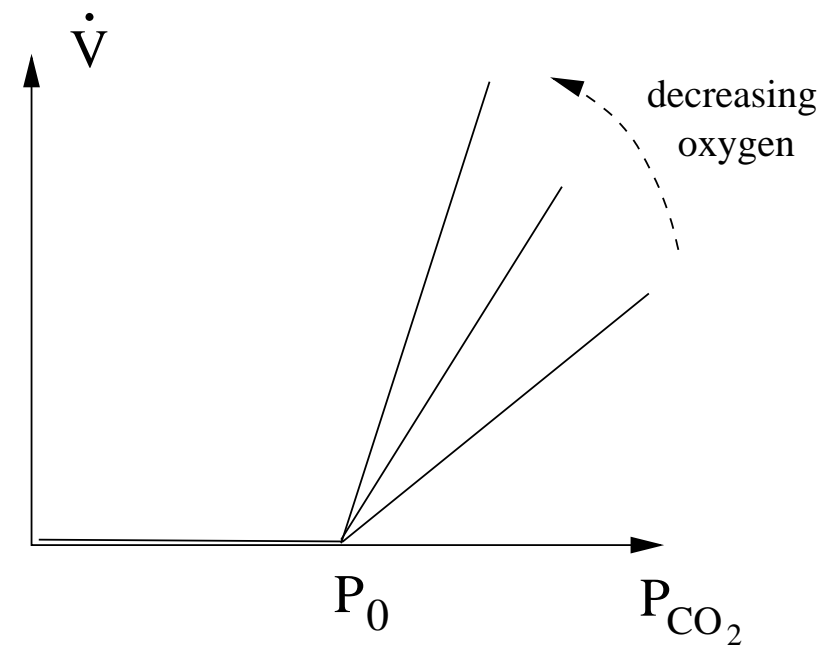

FiguRE 2. Schematic form of the peripheral ventilation controller. The central chemoreceptor has a similar response (with respect to $\mathrm{H}^{+}$), but without the oxygen dependence. In reality, the switch point at $P_{0}$ also decreases as oxygen levels decrease.

The central chemoreceptors in the brainstem have a similar response curve, but they actually respond to $\mathrm{H}^{+}$ions, rather than $\mathrm{CO}_{2}$, and this is mediated by $\mathrm{CO}_{2}$ through the acid-base buffering reaction

$$
\mathrm{H}_{2} \mathrm{O}+\mathrm{CO}_{2} \underset{k_{-1}}{\stackrel{k_{1}}{\rightleftharpoons}} \mathrm{HCO}_{3}^{-}+\mathrm{H}^{+}
$$

which is instrumental in maintaining the body's $\mathrm{pH}$ level. The point is that the blood-brain barrier is relatively impermeable to $\mathrm{H}^{+}$, but very permeable to $\mathrm{CO}_{2}$; therefore the central controller responds indirectly to blood $\mathrm{CO}_{2}$ at the brain by its rapid diffusion to the cerebro-spinal fluid, reaction with water to form hydrogen ions, with a consequent effect on the ventilatory controller. As is commonly done, in this paper we ignore details of this (rapid) reaction scheme, and simply suppose that the central controller responds directly to $\mathrm{CO}_{2}$ levels in the brain, in a manner similar to figure 2. Some comments on this assumption are made in the conclusions.

The part of the Grodins model described above is similar to that of Khoo et al. [17], as also expounded by Batzel and Tran [1]. In Batzel and Tran's exposition, the model is as above, but also includes equations for alveolar and venous oxygen (as does the full Grodins model), but we omit a consideration of oxygen in the present discussion. 


\begin{tabular}{|c|c|c|c|}
\hline Symbol & Units & \multicolumn{2}{|c|}{ Typical value } \\
\hline & & Grodins & Batzel/Tran \\
\hline$D_{\mathrm{CO}_{2}}$ & $1(\mathrm{STPD}) \min ^{-1} \mathrm{~mm} \mathrm{Hg}^{-1}$ & $0.82 \times 10^{-5}$ & - \\
\hline$G_{C}$ & 1 (BTPS) $\min ^{-1} \mathrm{~mm} \mathrm{Hg}^{-1}$ & & 1.8 \\
\hline$I_{C}$ & $\mathrm{~mm} \mathrm{Hg}$ & & 37 \\
\hline$k$ & atm $\mathrm{mm} \mathrm{Hg}^{-1}$ & 0.0013 & \\
\hline$K_{\mathrm{L}}$ & l(BTPS) & 3 & 3.2 \\
\hline$K_{\mathrm{B}}$ & 1 & 1 & 0.9 \\
\hline$K_{\mathrm{T}}$ & 1 & 39 & $14.1^{b}$ \\
\hline$K_{\mathrm{CO}_{2}}$ & $\mathrm{l}(\mathrm{STPD}) \mathrm{l}^{-1} \mathrm{~mm} \mathrm{Hg}^{-1}$ & {$[0.005]$} & 0.0057 \\
\hline$K_{\mathrm{CSF}}$ & 1 & 0.1 & \\
\hline$M R_{\mathrm{BCO}_{2}}$ & $1(\mathrm{STPD}) \mathrm{min}^{-1}$ & 0.05 & 0.042 \\
\hline$M R_{\mathrm{TCO}_{2}}$ & $1(\mathrm{STPD}) \min ^{-1}$ & 0.182 & $0.178^{c}$ \\
\hline$P^{*}$ & $\mathrm{~mm} \mathrm{Hg}$ & 40 & 40 \\
\hline$Q_{B}^{*}$ & $1 \min ^{-1}$ & 0.75 & 0.8 \\
\hline$Q^{*}$ & $1 \min ^{-1}$ & 6 & 6 \\
\hline$V^{*}$ & $1(\mathrm{BTPS}) \mathrm{min}^{-1}$ & 5 & $3.6^{a}$ \\
\hline$V_{\mathrm{aB}}$ & 1 & 1.06 & 1.06 \\
\hline$\tilde{V}_{\mathrm{aB}}$ & 1 & 0.015 & 0.016 \\
\hline$\alpha_{\mathrm{CO}_{2}}$ & $1(\mathrm{STPD}) \mathrm{l}^{-1} \mathrm{~atm}^{-1}$ & 0.51 & \\
\hline$\tau_{\mathrm{aB}}$ & $\min (\mathrm{sec})$ & $0.18(11)$ & $0.2(12)$ \\
\hline$\tau_{\mathrm{aT}}$ & $\min (\mathrm{sec})$ & $0.32(19)$ & $0.32(19)$ \\
\hline$\tau_{\mathrm{vT}}$ & $\min (\mathrm{sec})$ & $0.59(35)$ & $0.42(25)$ \\
\hline$\tau_{\mathrm{vB}}$ & $\min (\mathrm{sec})$ & $0.11(7)$ & - \\
\hline 863 & mm Hg l(BTPS) l(STPD $)^{-1}$ & 863 & \\
\hline
\end{tabular}

TABLE 1. Representative parameter values for the Grodins model, as given by Grodins et al. [14] and Batzel and Tran [1]. For the latter values: ${ }^{a} E_{F} \dot{V}_{A}$ in their table $1 ;{ }^{b}$ total tissue volume minus brain volume; ${ }^{c}$ total tissue production minus brain production.

\section{Non-dimensionalisation}

The parameters of the model, as given essentially by Grodins et al. [14], are shown in table 1. The value of $K_{\mathrm{CO}_{2}}$ is taken from a local linear fit to their dissociation (i. e., solubility) curve. Typical observed values of ventilation, blood flow and arterial $\mathrm{CO}_{2}$ partial pressure are denoted by $V^{*}, Q^{*}$ and $P^{*}$ respectively; representative values are $51 \mathrm{~min}^{-1}, 6 \mathrm{l} \mathrm{min}^{-1}$ and $40 \mathrm{~mm} \mathrm{Hg}$. A typical value of the blood flow to the brain is $Q_{B}^{*}$, and is $0.75 \mathrm{l} \mathrm{min}{ }^{-1}$. We therefore define dimensionless blood flows $q, q_{B}$ and ventilation rate $v$ by

$$
Q=Q^{*} q, \quad Q_{B}=Q_{B}^{*} q_{B},
$$

and

$$
\dot{V}=V^{*} v
$$

The $\mathrm{CO}_{2}$ partial pressure in the blood is determined by a balance between metabolic production and its removal by ventilation. It is easy to solve (2.1) and (2.2) to find the steady state partial pressures in terms of (constant) ventilation $V^{*}$ and blood flows $Q^{*}$ and $Q_{B}^{*}$. These are

$$
P_{a}=P^{*},
$$

where

$$
P^{*}=\frac{863}{V^{*}}\left[M R_{\mathrm{TCO}_{2}}+M R_{\mathrm{BCO}_{2}}\right]
$$


where the conversion factor 863 is the same as that in (2.1). Then, in the steady state,

$$
\begin{aligned}
P_{v} & =P^{*}(1+\varepsilon), \\
P_{B} & =P^{*}(1+\varepsilon a), \\
P_{T} & =P^{*}(1+\varepsilon b),
\end{aligned}
$$

where

$$
\varepsilon=\frac{V^{*}}{863 K_{\mathrm{CO}_{2}} Q^{*}},
$$

and

$$
a=\left(\frac{Q^{*}}{Q_{B}^{*}}\right)\left(\frac{M R_{\mathrm{BCO}_{2}}}{M R_{\mathrm{TCO}_{2}}+M R_{\mathrm{BCO}_{2}}}\right), \quad b=\left(\frac{Q^{*}}{Q^{*}-Q_{B}^{*}}\right)\left(\frac{M R_{\mathrm{TCO}_{2}}}{M R_{\mathrm{TCO}_{2}}+M R_{\mathrm{BCO}_{2}}}\right) .
$$

Using the (Grodins) values in table 1, we find typical values of these dimensionless parameters to be

$$
a=1.72, \quad b=0.9, \quad \varepsilon=0.19 .
$$

In scaling the blood $\mathrm{CO}_{2}$ partial pressure, we note that $\mathrm{CO}_{2}$ levels in the body do not vary enormously, and this is manifested in (2.12) by the relatively small value of the parameter $\varepsilon$. For example, venous blood returning to the lungs may only have a $\mathrm{CO}_{2}$ partial pressure of $45 \mathrm{~mm} \mathrm{Hg}$. (The precise steady value using the values of $P^{*}$ and $\varepsilon$ in (2.15) and table 1 is $47.6 \mathrm{~mm} \mathrm{Hg.)} \mathrm{Thus} \mathrm{the} \mathrm{significant} \mathrm{scale} \mathrm{for} \mathrm{the}$ $\mathrm{CO}_{2}$ variables is their variation about this typical value. Therefore we non-dimensionalise the pressure variables by writing

$$
\begin{aligned}
& P_{\mathrm{aCO}_{2}}=P^{*}\left[1+\varepsilon p_{a}\right], P_{\mathrm{vCO}_{2}}=P^{*}\left[1+\varepsilon p_{v}\right], \\
& P_{\mathrm{BCO}_{2}}=P^{*}\left[1+\varepsilon p_{B}\right], P_{\mathrm{TCO}_{2}}=P^{*}\left[1+\varepsilon p_{T}\right], \\
& P_{\mathrm{CSFCO}_{2}}=P^{*}\left[1+\varepsilon p_{C}\right] .
\end{aligned}
$$

Finally we choose the dimensionless time scale

$$
t \sim \frac{K_{B}}{Q_{B}^{*}}
$$

which as we shall see is representative of the typical period of Cheyne-Stokes oscillations. The dimensionless form of the equations (2.1), (2.2) and (2.3) is then

$$
\begin{aligned}
\dot{p}_{a} & =\Lambda\left[q\left(p_{v}-p_{a}\right)-\left(1+\varepsilon p_{a}\right) v\right], \\
\dot{p}_{B} & =a+q_{B}\left[p_{a}\left(t-\tau_{\mathrm{aB}}^{*}\right)-p_{B}\right]-\nu\left(p_{B}-p_{C}\right), \\
\dot{p}_{T} & =s\left[b+\left(\frac{q-\delta q_{B}}{1-\delta}\right)\left\{p_{a}\left(t-\tau_{\mathrm{aT}}^{*}\right)-p_{T}\right\}\right], \\
\dot{p}_{C} & =\mu\left(p_{B}-p_{C}\right), \\
p_{v} & =p_{T}\left(t-\tau_{\mathrm{vT}}^{*}\right)+\frac{\delta q_{B}}{q}\left[p_{B}\left(t-\tau_{\mathrm{vB}}^{*}\right)-p_{T}\left(t-\tau_{\mathrm{vT}}^{*}\right)\right] .
\end{aligned}
$$

The new parameters appearing in these equations are defined as follows:

$$
\begin{aligned}
\delta=\frac{Q_{B}^{*}}{Q^{*}}, \quad \Lambda & =\frac{863 K_{\mathrm{CO}_{2}} Q^{*} K_{B}}{K_{L} Q_{B}^{*}}, \quad s=\frac{\left(Q^{*}-Q_{B}^{*}\right) K_{B}}{K_{T} Q_{B}^{*}}, \\
\mu & =\frac{D_{\mathrm{CO}_{2}} K_{B}}{K_{\mathrm{CSF}} k \alpha_{\mathrm{CO}_{2}} Q_{B}^{*}}, \quad \nu=\frac{D_{\mathrm{CO}_{2}}}{K_{\mathrm{CO}_{2}} Q_{B}^{*}} .
\end{aligned}
$$


Using the (Grodins) values in table 1, we find typical values of these dimensionless parameters to be

$$
\delta=0.13, \quad \Lambda=11.5, \quad s=0.18, \quad \mu=0.16, \quad \nu=2.2 \times 10^{-3} .
$$

Grodins et al. [14] give expressions for the delays (their equations 8.10-8.13) which are variable, depending on blood flow. Typical values of the delays are given in table 1. In this paper we will take blood flow, and thus also the delays, as being constant. In this case, the dimensionless (scaled as in (2.17)) delays are defined by

$$
\begin{aligned}
& \tau_{\mathrm{aB}}^{*}=\frac{1}{K_{B}}\left[\frac{\delta V_{a B}}{q}+\frac{\tilde{V}_{a B}}{q_{B}}\right], \\
& \tau_{\mathrm{aT}}^{*}=\frac{1}{K_{B}}\left[\frac{\delta V_{a T}}{q}+\frac{\delta \tilde{V}_{a T}}{q-\delta q_{B}}\right], \\
& \tau_{\mathrm{vB}}^{*}=\frac{1}{K_{B}}\left[\frac{V_{v B}}{q_{B}}+\frac{\delta \tilde{V}_{v B}}{q}\right], \\
& \tau_{\mathrm{vT}}^{*}=\frac{1}{K_{B}}\left[\frac{\delta V_{v T}}{q-\delta q_{B}}+\frac{\delta \tilde{V}_{v T}}{q}\right],
\end{aligned}
$$

where $V_{a B} \approx 1.062 \mathrm{l}, \tilde{V}_{a B} \approx 0.015 \mathrm{l}, V_{a T} \approx 1.062 \mathrm{l}, \tilde{V}_{a T} \approx 0.735 \mathrm{l}, V_{v T} \approx 2.94 \mathrm{l}, \tilde{V}_{v T} \approx 0.188 \mathrm{l}, V_{v B} \approx 0.06$ 1, $\tilde{V}_{v B} \approx 0.188 \mathrm{l}$ are blood vessel volumes. The numerical values are those given by Grodins et al. [14]. Typical values of the dimensionless delays, based on the estimates in table 1, are

$$
\tau_{\mathrm{aB}}^{*}=0.14, \quad \tau_{\mathrm{aT}}^{*}=0.24, \quad \tau_{\mathrm{vB}}^{*}=0.08, \quad \tau_{\mathrm{vT}}^{*}=0.44 .
$$

Provided the blood flows $q$ and $q_{B}$ are prescribed, as well as the ventilation function $v,(2.18)$ provides a set of four differential equations with four separate delays for the $\mathrm{CO}_{2}$ partial pressures in the various compartments of the model. Evidently the steady state, with $v=q=q_{B}=1$, is $p_{a}=0, p_{v}=1, p_{B}=a$, $p_{T}=b$, since satisfaction of $(2.18)_{4}$ is then automatic, as

$$
1=\delta a+(1-\delta) b
$$

\section{The Mackey-Glass model}

The model proposed by Mackey and Glass [20] is a one compartment model, and can be motivated by the previously stated observation that the $\mathrm{CO}_{2}$ partial pressure is relatively uniform throughout the body. Denoting this one partial pressure as $P$, a single conservation equation which describes the balance between production and ventilation is just

$$
K \frac{d P}{d t}=M-A P \dot{V},
$$

in which $K$ is compartment volume and $M$ is total metabolic production rate; $A$ is a suitable numerical conversion factor, which may be necessary to include when (3.1) is derived under certain assumptions from the Grodins model. The difficulty with this model is exemplified by the choice of ventilator control. $\dot{V}$ is taken as a function of $P(t-\tau)$, where $\tau$ is the transit delay between lung and brain: this represents the central chemoreceptor's response to $\mathrm{CO}_{2}$, but it conceptually indicates that the blood at the brain is somehow not part of the compartment, whereas intrinsically the whole body is being treated as a single compartment. 
The model thus makes better conceptual sense if the brain is thought of as a shunt which produces little $\mathrm{CO}_{2}$, as indicated in figure 3. With this in mind, we would interpret the quantities $K$ and $M$ as

$$
K=K_{T}, \quad M=\frac{M R_{\mathrm{TCO}_{2}}}{K_{\mathrm{CO}_{2}}}
$$

alternatively, we might add the brain volume and production rate into the above quantities.

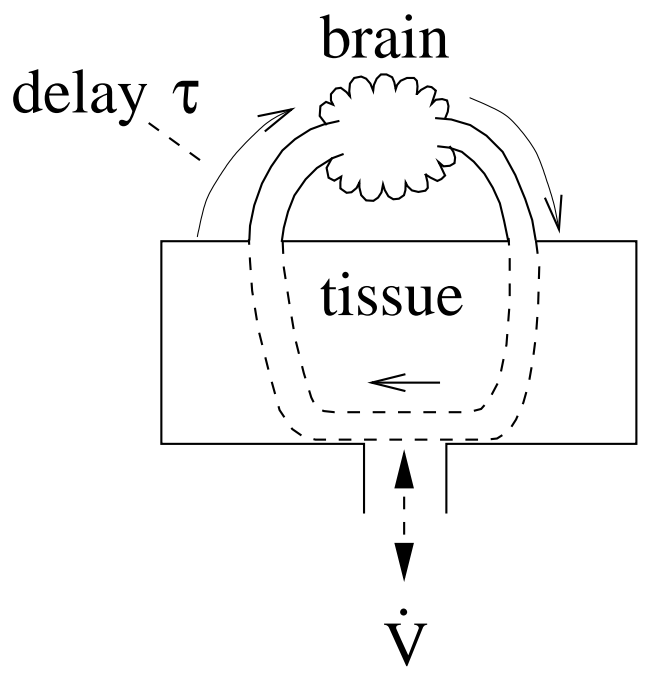

Figure 3. A possible compartment scheme for the Mackey-Glass model. The tissues represent the compartment, produce $\mathrm{CO}_{2}$, and are ventilated at a rate $\dot{V}$ by the arterial blood flow, which is shunted through the brain.

This is not the only interpretation of the Mackey-Glass model in terms of a single compartment. We can take the lungs as the single compartment, as indicated in figure 4. The tissues supply $\mathrm{CO}_{2}$ at the rate $M$ as before (with no delay), and the lungs are ventilated as before. As with the Grodins model, we suppose that alveolar and arterial gas concentrations are the same, which is a good approximation. In this second interpretation, we would choose

$$
K=A K_{L}, \quad M=\frac{M R_{\mathrm{TCO}_{2}}}{K_{\mathrm{CO}_{2}}} .
$$

(The conversion factor $A$ is necessary for consistency with the Grodins model.) We will consider both possibilities in our discussion.

There is a unique steady state of the Mackey-Glass model, if $\dot{V}$ is a monotonically increasing function of $P(t-\tau)$. If $\dot{V}=V^{*}$ and $P=P^{*}$ at this steady state, then $M=A P^{*} V^{*}$. The stability of the steady state is determined by writing

$$
P=P^{*}+\tilde{P},
$$

and linearising (3.1), by virtue of the supposition that $\tilde{P} \ll P^{*}$, to obtain

$$
K \frac{d \tilde{P}}{d t}=-A V^{*} \tilde{P}-A P^{*} G \tilde{P}_{\tau}
$$

where $\tilde{P}_{\tau}=\tilde{P}(t-\tau)$, and

$$
V^{*}=\dot{V}\left(P^{*}\right)=M / A P^{*}, \quad G=\dot{V}^{\prime}\left(P^{*}\right),
$$




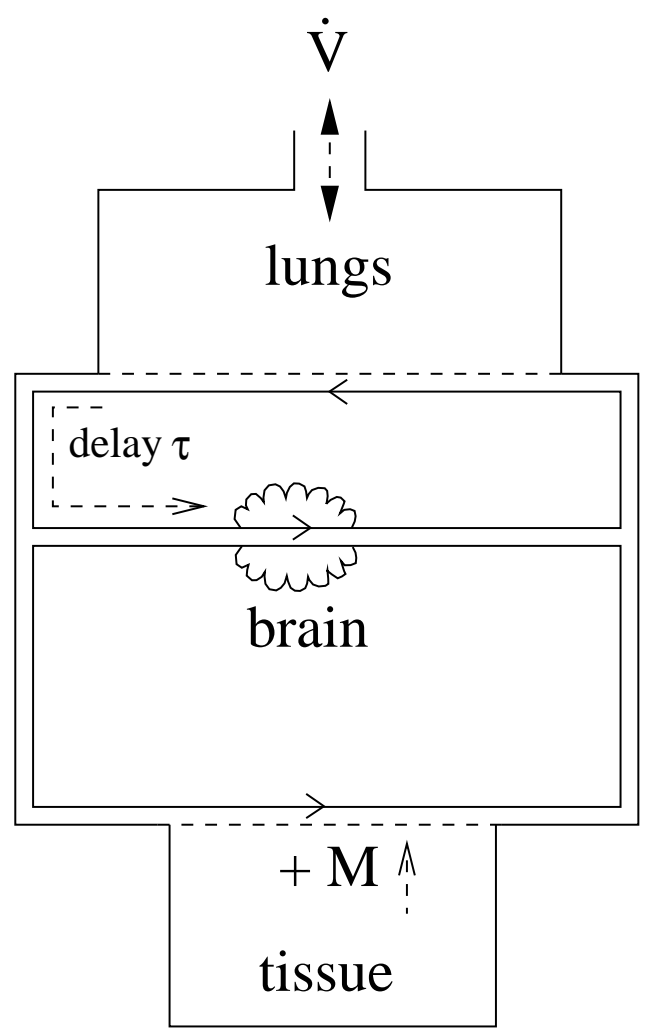

Figure 4. A compartment scheme for the Mackey-Glass model in which the lungs represent the compartment. Alveolar $\mathrm{CO}_{2}$ is produced by the tissues and removed by ventilation, and there is a single delay in shunting the blood to the brain.

this latter quantity being the controller gain. Solutions to (3.5) of the form $\exp (\sigma t)$ exist, provided

$$
K \sigma=-\left[A V^{*}+A P^{*} G e^{-\sigma \tau}\right] .
$$

The stability characteristics depend on the two dimensionless parameters $B=A V^{*} \tau / K$ and $\gamma=$ $A P^{*} G \tau / K$, and then $\sigma=\lambda / \tau$, where

$$
\lambda=-B-\gamma e^{-\lambda} .
$$

When $B$ is small (as here), then one can show that oscillatory (Hopf) instability occurs if

$$
\frac{A \tau P^{*} G}{K} \gtrsim \frac{\pi}{2}
$$

and the resulting oscillations have period $\approx 4 \tau$. We discuss the applicability of this result in the discussion. More generally, instability occurs if $\gamma>\gamma_{1}(B)$, where $\gamma_{1}(B)$ is a monotonically increasing function, as shown in figure 5 .

\subsection{The Mackey-Glass-Grodins limit}

We now wish to enquire under what circumstance the Mackey-Glass model emerges as a parametric limit of the Grodins model. The way in which this can occur is if two of the three rate equations are fast and the other is relatively slow. We have provided two ways in which the Mackey-Glass model can be interpreted as a compartment model, and we discuss each of these in turn. 


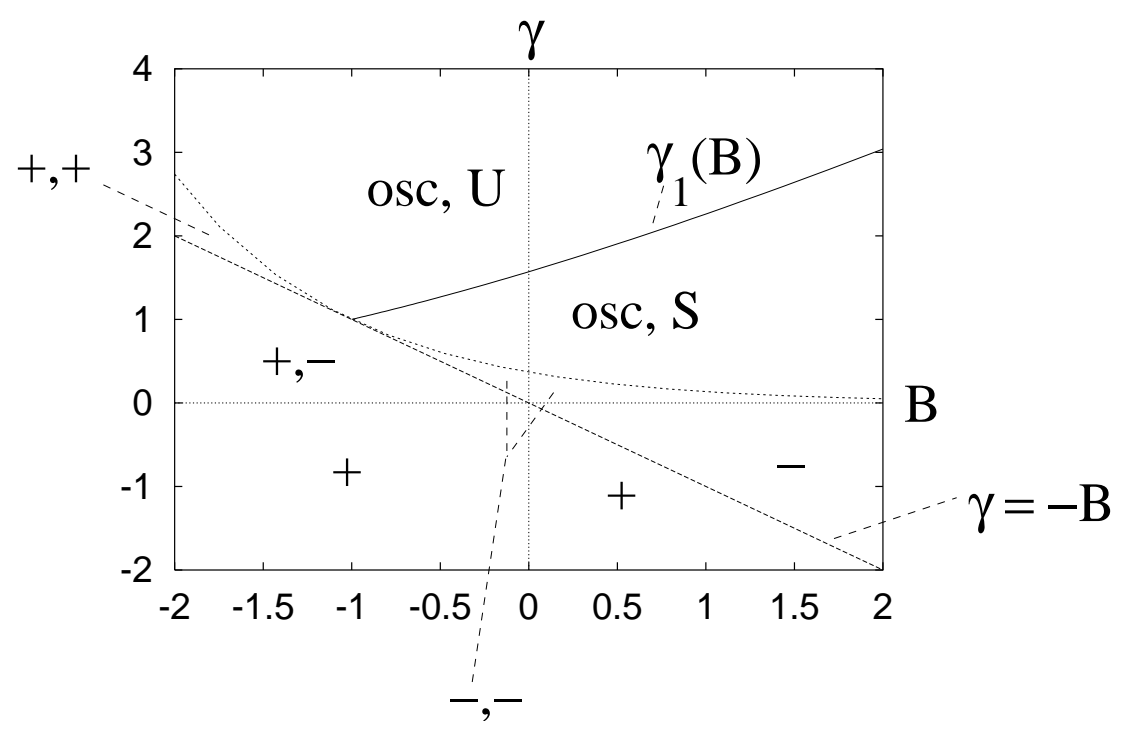

FiguRE 5. Stability map for the solutions of (3.8). The curves demarcate the behaviours of the two roots involved in the transition to instability. 'osc' means complex conjugate, and ' $\mathrm{U}$ ' is unstable, ' $\mathrm{S}$ ' is stable; ' + ' and '-' refer to the signs of real roots; where only one sign is indicated, the other root has disappeared by tending to infinity. The diagram indicates that oscillatory stability occurs for $B, \gamma>0$ when $\gamma>\gamma_{1}(B)$.

\subsubsection{Case 1: tissue compartment}

In view of the necessary interpretation in case 1 of $K$ in (3.1) as tissue volume, it seems we must have the tissue equation $(2.1)_{3}$ as the slowest equation. If we suppose all delays except the shunt delay $\tau_{\mathrm{aB}}$ are zero, and that $Q_{B} \ll Q$, then (2.2) will imply that

$$
P_{\mathrm{vCO}_{2}} \approx P_{\mathrm{TCO}_{2}} .
$$

Next, suppose $M R_{\mathrm{BCO}_{2}}$ is small, and that the $P_{\mathrm{BCO}_{2}}$ equation is fast: then

$$
P_{\mathrm{BCO}_{2}} \approx P_{\mathrm{aCO}_{2}}\left(t-\tau_{\mathrm{aB}}\right) .
$$

Finally, if the $P_{\mathrm{aCO}_{2}}$ equation is fast, then

$$
\dot{V} P_{\mathrm{aCO}_{2}} \approx 863 K_{\mathrm{CO}_{2}} Q\left[P_{\mathrm{vCO}_{2}}-P_{\mathrm{aCO}_{2}}\right] .
$$

Substituting this into $(2.1)_{3}$, and using also the fact that if $\varepsilon$ in (2.13) is small, then (3.12) also implies $P_{\mathrm{vCO}_{2}} \approx P_{\mathrm{aCO}_{2}}$, we derive the approximate equation for $P=P_{\mathrm{TCO}_{2}}$ in the Mackey-Glass form

$$
K \dot{P}=M-A P \dot{V},
$$

where $K$ and $M$ are as defined in (3.2), and

$$
A=\frac{1}{863 K_{\mathrm{CO}_{2}}}=0.23 \mathrm{l}(\mathrm{BTPS})^{-1} .
$$

If $\dot{V}$ depends on $P_{\mathrm{BCO}_{2}}$, then because of (3.11), we have

$$
\dot{V}=\dot{V}\left[P\left(t-\tau_{\mathrm{aB}}\right)\right],
$$

as in the Mackey-Glass model. 


\subsubsection{Case 2: lung compartment}

In the second case, we must suppose that the arterial $P_{\mathrm{aCO}_{2}}$ equation is the slowest. We again suppose that all delays except the shunt delay $\tau_{\mathrm{aB}}$ are zero, and that $Q_{B} \ll Q$, and also that brain metabolic $\mathrm{CO}_{2}$ production is small. Then again we obtain (3.10) and (3.11). Now we suppose that the tissue equation is fast, so that (using (3.10))

$$
Q\left[P_{\mathrm{vCO}_{2}}-P_{\mathrm{aCO}_{2}}\right] \approx M,
$$

where $M$ is as given in (3.3). With $P=P_{\mathrm{aCO}_{2}}$, we then obtain the Mackey-Glass equation (3.1), with $K, M$ and $A$ given by (3.3) and (3.14).

\subsubsection{Validity}

There may be other ways of reducing the Grodins model to the Mackey-Glass model, but it seems that these two are the most direct. Let us examine the parametric basis on which the reduction rests. Consulting (2.18), we require for both cases 1 and 2, that the non-controlling delays are negligible:

$$
\tau_{v B}^{*}, \tau_{v T}^{*}, \tau_{a T}^{*} \ll 1
$$

we require the blood flow and brain metabolic production rate to be small:

$$
a, \delta, \ll 1 .
$$

The first of these is not, in fact, essential. For case 1 (tissue compartment), we require the brain and arterial equations to be fast:

$$
s \ll 1, \quad s \ll \Lambda \quad \text { (case } 1) .
$$

On the other hand for case 2 (lung compartment), we require tissue and brain equations to be fast, and this requires

$$
\Lambda \ll 1, \quad \Lambda \ll s \quad(\text { case } 2) .
$$

\section{Reducing the Grodins model}

The numerical estimates of the parameters in (2.15), (2.20) and (2.22) are typical values, but there may be a good degree of variability from individual to individual. As is intuitively obvious, the delays to and from the tissues are actually larger than the delays to and from the brain, so clearly the neglect of these delays is inadmissible. However, it is possible that the Mackey-Glass reduction could still be useful, if these delays have no destabilising effect of their own.

Typical values for $\Lambda$ and $s$ in (2.18) are $\Lambda \approx 11.5, s \approx 0.18$. We see that case 2 is immediately ruled out, since the actual $s \ll 1 \ll \Lambda$ contradicts $(3.20)$.

However, case 1 is apparently possible. The difficulty here is that the time scale we have chosen is

$$
t \sim \frac{K_{B}}{Q_{B}^{*}} \approx 80 \mathrm{~s},
$$

which is comparable to the observed period of Cheyne-Stokes respiration. The time scale for tissue response is $K_{B} / Q_{B}^{*} s \approx 7.4 \mathrm{~min}$. Thus the time scale of the tissue equation is much too long to be able to explain the minute scale oscillations. Equivalently, the value of the critical parameter $\gamma=A \tau P^{*} G / K$ in (3.9) is about 0.09 for case 2 , and instability is not feasible. Instability is feasible for the lung compartment model, but as we have just seen, this is in no way tenable as a reduction from the Grodins model.

In order to find a suitable reduction of the Grodins model which describes oscillations on a time scale of a minute, we keep $t=O(1)$ in (2.18). Since $s \ll 1$ and $\Lambda \gg 1$, the tissue equation is slow and the arterial equation is fast. Using additionally the approximations $\delta, \varepsilon \ll 1$, we have

$$
\begin{aligned}
p_{v} & \approx p_{T}\left(t-\tau_{\mathrm{vT}}^{*}\right), \\
q\left(p_{v}-p_{a}\right) & \approx v .
\end{aligned}
$$


Because $s$ is small, $p_{T}$ and thus also $p_{v}$ is slowly varying over a time scale of $O(1 / s) \approx 5$; therefore we can average $(2.18)_{3,4}$ to obtain ( $\operatorname{since} \tau_{\mathrm{vT}}^{*}, \tau_{\mathrm{aT}}^{*} \ll 1 / s$ )

$$
\begin{aligned}
p_{v} & \approx p_{T}, \\
\dot{p}_{T} & \approx s\left[b+q\left(p_{a}-p_{v}\right)\right] .
\end{aligned}
$$

Using $(4.2)_{2}$, this last equation can be written

$$
\dot{p}_{T} \approx s[b-v],
$$

and shows that tissue $\mathrm{CO}_{2}$ builds up until the average ventilation balances the metabolic production rate,

$$
\bar{v}=b,
$$

where the overbar denotes the time average. For stationary or oscillatory variations in $p_{a}$ and $p_{B}$, we can take $p_{T}$ and $p_{v}$ to be approximately constant, and such that (4.5) applies. Now $(4.2)_{2}$ implies that $p_{a}=p_{v}-(v / q)$, and thus, with $v=v\left(p_{B}\right),(2.18)_{3}$ implies

$$
\dot{p}_{B} \approx a+q_{B}\left[p_{v}-\frac{v\left\{p_{B}\left(t-\tau_{\mathrm{aB}}^{*}\right)\right\}}{q\left(t-\tau_{\mathrm{aB}}^{*}\right)}-p_{B}\right],
$$

where $p_{v}$ is a constant, to be chosen so that (4.5) applies. This, finally, is the version of the Mackey-Glass equation which forms a consistent reduction of the Grodins model.

If we make the further assumption that blood flow is constant $\left(q=q_{B}=1\right)$, or at least does not vary greatly, then (4.6) simplifies further, and can be reduced to the single equation for $p_{B}$ :

$$
\dot{p}_{B} \approx 1+\bar{p}_{B}-v\left\{p_{B}\left(t-\tau_{\mathrm{aB}}^{*}\right)\right\}-p_{B} .
$$

In writing this, we have put $b=1$, consistent with the neglect of terms of $O(\delta)$ in $(2.23)$, and have used the time average of (4.6) to define $p_{v}$ in terms of $\bar{p}_{B}$. The equation (4.7) may serve as the simplest paradigm in which the onset of periodic breathing can be understood. It is indeed a Mackey-Glass type equation, but with the fundamental difference that the mean ventilation is controlled to be constant.

\subsection{Choice of controller function}

We initially suppose that the controller function $\dot{V}$ depends only on $P_{\mathrm{BCO}_{2}}$. Typical ventilatory responses in hypercapnia are linear, of the form

$$
\dot{V}=G_{C}\left[P_{\mathrm{BCO}_{2}}-P_{0}\right]_{+},
$$

with apnea occurring below the threshold value $P_{0}$. Since by choice, $\dot{V}=V^{*}$ when $P_{\mathrm{BCO}_{2}}=P^{*}(1+\varepsilon a)$, and since $P^{*}$ is related to $V^{*}$ by (2.11), it follows, using the definitions of $\varepsilon$ and $a$ in (2.13) and (2.14), that $V^{*}$ is determined by the unique positive value which satisfies these constraints, that is,

$$
V^{*}=\frac{1}{2}\left[-G_{C} \hat{P}+\left\{\left(G_{C} \hat{P}\right)^{2}+4 \times 863 G_{C}\left(M R_{\mathrm{TCO}_{2}}+M R_{\mathrm{BCO}_{2}}\right)\right\}^{1 / 2}\right],
$$

where

$$
\hat{P}=P_{0}-\frac{M R_{\mathrm{BCO}_{2}}}{Q_{B}^{*} K_{\mathrm{CO}_{2}}} .
$$

Then the dimensionless ventilation controller is given by

$$
v\left(p_{B}\right)=\left[1+\Gamma\left(p_{B}-a\right)\right]_{+},
$$

where the dimensionless gain is

$$
\Gamma=\frac{G_{C}\left(M R_{\mathrm{TCO}_{2}}+M R_{\mathrm{BCO}_{2}}\right)}{V^{*} K_{\mathrm{CO}_{2}} Q^{*}} .
$$

According to the values in table $1, \Gamma \approx 2.78$. 


\subsection{Linear stability}

We write

$$
p_{B}=a+p,
$$

so that $p=0$ is the rest state. Denoting also the dimensionless delay as $\tau_{\mathrm{aB}}^{*}=\tau$, and denoting $p(t-\tau) \equiv$ $p_{\tau},(4.7)$ becomes

$$
\dot{p} \approx 1+\bar{p}-\left[1+\Gamma p_{\tau}\right]_{+}-p .
$$

For small (oscillatory) perturbations $p \ll 1$, we may take $\bar{p}=0$, and (4.14) is just

$$
\dot{p} \approx-\Gamma p_{\tau}-p,
$$

whose solutions $p=e^{\sigma t}$ satisfy

$$
\sigma=-1-\Gamma e^{-\sigma \tau}
$$

comparable to (3.8), so that instability occurs for

$$
\Gamma>\frac{\gamma_{1}(\tau)}{\tau}
$$

and $\gamma_{1}$ is shown in figure 5 .

Since $\tau=\tau_{\mathrm{aB}}^{*} \approx 0.14$ is small, $\gamma_{1} \approx \pi / 2$. Thus instability occurs for

$$
\Gamma \gtrsim \Gamma_{c}=\frac{\pi}{2 \tau_{\mathrm{aB}}^{*}} \approx 11.2
$$

with dimensionless period $P \approx 4 \tau_{\mathrm{aB}}^{*}$. (The exact value of $\Gamma_{c}$ from a computation of $\gamma_{1}(\tau)$ is 11.865 when $\tau=0.14$.) In terms of dimensional quantities, the oscillation period is approximately

$$
t_{p} \approx \frac{4 V_{\mathrm{aB}}}{Q_{B}}=4 \tau_{\mathrm{aB}}
$$

and the instability criterion can be written, using $\tau_{\mathrm{aB}}^{*}=\tau_{\mathrm{aB}} Q_{B}^{*} / K_{B},(2.11)$ and (3.14),

$$
\left(\frac{A \tau_{\mathrm{aB}} P^{*} G_{C}}{K_{B}}\right) \frac{Q_{B}^{*}}{Q^{*}} \gtrsim \frac{\pi}{2}
$$

and this can be compared to (3.9).

\section{Stability criteria}

We have shown that an instability criterion for all the simplified models we have discussed can be written in the form of (3.9), that is

$$
\tau P^{*} G_{C} \gtrsim \frac{\pi K}{2 A},
$$

but with differing choices of compartment volume $K$. For the Mackey-Glass tissue compartment model, case 1 , this is (see $(3.2)$ )

$$
K=K_{T} \quad \text { (Mackey-Glass tissue compartment); }
$$

for the Mackey-Glass lung compartment model, case 2, (see (3.3))

$$
K=A K_{L} \quad \text { (Mackey-Glass lung compartment); }
$$


while for the reduced Grodins model (see (4.20)) we have

$$
K=\frac{K_{B} Q^{*}}{Q_{B}^{*}} \quad \text { (reduced Grodins). }
$$

We now compare these criteria, and also the Mackey-Glass (5.1) model itself, using values of $K$ inferred from Mackey and Glass [20].

Using the normal values $A=0.23 \mathrm{l}$ (BTPS) $^{-1}, \tau=0.18 \mathrm{~min}, P^{*}=40 \mathrm{~mm} \mathrm{Hg}, G_{C}=1.8 \mathrm{l}(\mathrm{BTPS})$ $\mathrm{min}^{-1} \mathrm{~mm} \mathrm{Hg}^{-1}$, we find $G_{C} P^{*} \tau=13$ l(BTPS), while for the three cases listed above, we have

$$
\frac{\pi K}{2 A} \approx 6.83 K=\left\{\begin{array}{cl}
266 & \text { (Mackey-Glass tissue compartment) } \\
4.7 & \text { (Mackey-Glass lung compartment) } \\
54.6 & \text { (reduced Grodins) }
\end{array}\right.
$$

also in units of 1 (BTPS).

Mackey and Glass do not give explicit values for $K, M$ and $A$, but rather give values for $\lambda=M / K$ and $\alpha=A / K$, as well as values for $P^{*}(40 \mathrm{~mm} \mathrm{Hg}), V^{*}\left(71 \mathrm{~min}^{-1}\right)$ and $G_{C}\left(4 \mathrm{l} \mathrm{min}^{-1} \mathrm{~mm} \mathrm{Hg}^{-1}\right)$, the latter two being noticeably higher than the values used here. In the steady state, these are related by $\alpha=A / K=\lambda / P^{*} V^{*}$, and thus we find (using $\lambda=6 \mathrm{~mm} \mathrm{Hg} \mathrm{min}^{-1}$ )

$$
\frac{\pi K}{2 A} \approx 73.3 \mathrm{l} \text { (BTPS) (original Mackey-Glass). }
$$

This is comparable to the last value in (5.5), so that in fact the Grodins instability criterion is similar to that derived by Mackey and Glass. However the normal Mackey-Glass value of $G_{C} P^{*} \tau=40$ l(BTPS) is a good deal higher than our estimate of 13 l(BTPS), since as well as the higher gain, they take the delay to be $\tau=0.25 \mathrm{~min}$ ( 15 seconds). Thus the Mackey-Glass choice of parameters is already nearer instability, and a doubling of the gain (in stroke) or a halving of the blood flow (in heart failure) can cause oscillations.

\subsection{The Francis model}

An interesting form of Mackey-Glass model has been developed by Francis et al. [13] and Manisty et al. [21], in particular because in the earlier of these two papers, the results of the model are compared to clinical data - with good results. The Francis model can be written in the form

$$
K_{L} \dot{c}=M-\dot{V} c-\beta Q\left(c-c^{*}\right)
$$

in which $K_{L}$ is alveolar volume, $c$ is alveolar $\mathrm{CO}_{2}$ fraction, and the last term represents a transfer from the lung into extrapulmonary stores (i.e., tissues); $\dot{V}$ is alveolar ventilation and is a function of the delayed concentration $c_{\tau}, Q$ is cardiac output, and $\beta$ is the solubility of $\mathrm{CO}_{2}$ in blood; $c^{*}$ is the steady state, and the value in the tissues is assumed constant.

Comparing this to $(2.1)_{1}$, we see that this model is precisely equivalent to the Grodins model, with the additional assumptions that $P_{\mathrm{vCO}_{2}}=P_{\mathrm{TCO}_{2}}$ and that $P_{\mathrm{TCO}_{2}}$ is approximately constant: both assumptions which we have shown to be true! In addition, however, there is a crucial assumption that $P_{\mathrm{BCO}_{2}}=P_{\mathrm{aCO}_{2}}(t-\tau)$, but this is inaccurate, as can be seen in (2.18). The Francis assumption is equivalent to the parametric limit $s \ll \Lambda \ll 1$.

Linearising (5.7), we obtain, as did Francis et al. [13], (3.8), with corresponding definitions

$$
\gamma=\frac{\tau P^{*} G_{C}}{K_{L}}, \quad B=\left(V^{*}+\frac{Q^{*}}{A}\right) \frac{\tau}{K_{L}},
$$

and instability occurs for $\gamma>\gamma_{1}(B)$. The variables used by Francis et al. are $\bar{C} S=G_{C} P^{*}, \delta=\tau$, $V_{L}=K_{L}, \overline{\dot{V}}_{A}=V^{*}, \beta=1 / A($ see $(2.1)$ and $(3.14))$, and $\overline{\dot{Q}}=Q^{*}$. 


\begin{tabular}{|l|c|c|}
\hline \multicolumn{1}{|c|}{ Parameter } & Grodins/Batzel & Francis \\
\hline$C S=G_{C} P^{*}\left[\mathrm{l}(\mathrm{BTPS}) \mathrm{min}^{-1}\right]$ & 72 & 25.2 \\
$\dot{Q}=Q^{*}\left[1 \mathrm{~min}^{-1}\right]$ & 6 & $4.2^{a}$ \\
$V_{L}=K_{L}[\mathrm{l}(\mathrm{BTPS})]$ & 3 & 3.2 \\
$\dot{V}_{A}=V^{*}\left[\mathrm{l}(\mathrm{BTPS}) \mathrm{min}^{-1}\right]$ & 5 & - \\
$\beta=1 / A\left[1(\mathrm{BTPS}) \mathrm{l}^{-1}\right]$ & $4.35^{b}$ & 5 \\
$\delta=\tau[\mathrm{min}]$ & 0.18 & 0.3 \\
\hline
\end{tabular}

TABle 2. Parameter values as used in table 1 and as found by Francis et al. [13] ( ${ }^{a}$ assuming a $70 \mathrm{~kg}$ person; ${ }^{b}$ from (3.14)).

The parameter values found for normal control patients are compared with the Grodins values of table 1 in table 2. The corresponding normal values of $\gamma$ and $B$ are

$$
\begin{aligned}
& \gamma=2.36 \text { (Francis), } 4.32 \text { (Grodins), } \\
& B=2.44 \text { (Francis), } 1.87 \text { (Grodins). }
\end{aligned}
$$

Despite the seeming inappropriateness of the analysis, the Francis theory shows good agreement with clinical data (their figure 3). It is not clear whether the reduced Grodins prediction (4.20) might also fit the data. To get some idea of this, note that at large values of $B, \gamma_{1}(B) \approx B$, and if we use this, and also neglect $V^{*} \approx 5$ l(BTPS) $\mathrm{min}^{-1}$ in comparison with $Q^{*} / A \approx 26$ l(BTPS) $\mathrm{min}^{-1}$, then the Francis instability criterion is roughly

$$
\frac{A G_{C} P^{*}}{Q^{*}} \gtrsim 1
$$

and this can be compared with the reduced Grodins criterion, which can be written, taking

$$
\tau_{\mathrm{aB}} \approx \frac{V_{\mathrm{aB}}}{Q^{*}},
$$

as

$$
\frac{A G_{C} P^{*}}{Q^{*}} \gtrsim \frac{\pi K_{B} Q^{*}}{2 V_{\mathrm{aB}} Q_{B}^{*}},
$$

which is of similar form, though the right hand side of (5.12) is normally much larger than one. The Francis model is more susceptible to instability because it is essentially a Mackey-Glass lung compartment model.

Francis et al. measured their values of the delay by assessing response time to step changes of inhaled $\mathrm{CO}_{2}$, and found values as large as 40 seconds. If we go back to (2.5) and assess the values of $V_{\mathrm{aB}}$ and $\tilde{V}_{\mathrm{aB}}$ in table 1 , we can see that $V_{\mathrm{aB}}$ represents mostly heart and pulmonary circulation volume (which is typically $16 \%$ of blood volume ([16], figure $14-1$ ), while $\tilde{V}_{\mathrm{aB}}$ is the much smaller arterial volume from the aorta to the brain. Thus the Grodins schematic figure 1 is somewhat misleading in respect of the instrument of delay, which as regards passage to the chemoreceptors is actually due to transit through the pulmonary and coronary parts of the circulation, and this raises questions as to whether the Grodins model itself deals with the different compartments in the right way.

\subsection{Acid control}

At this point, we reconsider the notion that the central chemoreceptor actually responds to acid, i.e., $\mathrm{H}^{+}$concentration. As discussed earlier, acid-base buffering described by the reaction (2.7) ties the acid concentration to that of $\mathrm{CO}_{2}$, and we suppose therefore that in this case we can take the ventilatory

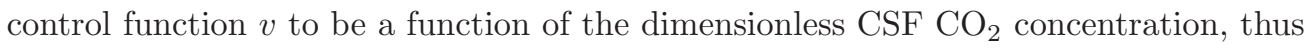

$$
v=v\left(p_{C}\right)=\left[1+\Gamma\left(p_{C}-a\right)\right]_{+},
$$


and we thus consider the reduced Grodins model

$$
\begin{aligned}
& \dot{p}_{B}=1+\bar{p}_{B}-v\left\{p_{C}(t-\tau)\right\}-p_{B}, \\
& \dot{p}_{C}=\mu\left(p_{B}-p_{C}\right) .
\end{aligned}
$$

We might expect that the extra buffering of the blood-brain barrier might contribute to instability, since in some sense it increases the delay between the lungs and the controller. To examine this, we linearise (5.14) by first writing $p_{B}=a+p, p_{C}=a+q$, and then seek solutions in the form $p=B e^{\sigma t}$, $q=C e^{\sigma t}$. Carrying out the algebra, we find that with the definitions

$$
\lambda=\sigma \tau, \quad m=\mu \tau, \quad g=\Gamma \tau,
$$

$\lambda$ satisfies the transcendental equation

$$
\lambda=-\tau-\frac{m g}{\lambda+m} e^{-\lambda}
$$

It is straightforward to analyse the solutions of this equation. There are a denumerable set of roots which occur as complex conjugates, and which have a limit point at infinity, with $\operatorname{Re} \lambda<0$ as $\lambda \rightarrow \infty$. When $g$ is small, all solutions have $\operatorname{Re} \lambda<0$. Instability can only occur as a Hopf bifurcation, when $\lambda= \pm i \Omega$, and then $\Omega$ satisfies

$$
\tan \Omega=\frac{\Omega(\tau+m)}{\Omega^{2}-\tau m},
$$

and the corresponding values of $g$ are

$$
g=\frac{\Omega}{\sin \Omega}\left(\frac{\tau+m}{m}\right) .
$$

(5.17) has a denumerable sequence of (positive, without loss of generality) roots $0<\Omega_{1}<\Omega_{2} \ldots$, and we denote the corresponding values of $g$ as $g_{1}, g_{2}$, etc. Evidently $g_{i}=g_{i}(\tau, m)$, and the $g_{i}$ form an increasing sequence. Thus instability occurs for $g>g_{1}(\tau, m)$.

The limit $m \rightarrow \infty$ reduces the model back to (4.7), but in fact an appropriate value is $m=0.02$, using the values of $\mu$ and $\tau=\tau_{\mathrm{aB}}^{*}$ in (2.20) and (2.22), and so the more appropriate limit is $m \rightarrow 0$. For $\tau m>\frac{1}{4} \pi^{2}, \Omega_{1} \in\left(\frac{1}{2} \pi, \pi\right)$, while for $\tau m<\frac{1}{4} \pi^{2}, \Omega_{1} \in\left(0, \frac{1}{2} \pi\right)$. For values $m=0.02, \tau=0.14$, we take $m \ll \tau \ll 1$, and then we can approximate

$$
\Omega_{1} \approx \sqrt{\tau}, \quad g_{1} \approx \frac{\tau}{m} .
$$

Instability thus occurs if

$$
\Gamma \tau \gtrsim \frac{1}{\mu}
$$

or

$$
\tau P^{*} G_{C} \gtrsim \frac{\pi K}{2 A}
$$

where

$$
K=\frac{2}{\pi} \frac{K_{B} Q^{*}}{Q_{B}^{*} \mu} .
$$

With $\mu=0.16$ this is 3.98 times the reduced value (5.4), thus

$$
\frac{\pi K}{2 A}=217.2 \quad \text { (reduced Grodins-CSF). }
$$

So in fact, the CSF buffering dramatically increases the stability. In fact, the limit $\mu \ll 1$ would imply, as for the tissue $\mathrm{CO}_{2}$ concentration, that $p_{C} \approx$ constant, and it is for this reason that the slow release of $\mathrm{CO}_{2}$ to the CSF enhances stability. 


\section{Conclusions}

It is possible to derive the Mackey-Glass model from the more realistic Grodins model in two ways. One of these would generally lead to instability (the lung compartment model), but it is not a valid reduction of the Grodins model as it requires $\Lambda \ll 1$ and $\Lambda \ll s$, neither of which is accurate (since $\Lambda \approx 11.5, s \approx 0.18$ ). The other (tissue compartment) model does represent a valid reduction of the Grodins model, but instability is not realistically attainable within this approximation. We conclude that the Mackey-Glass model is not a valid representation of the multi-compartment Grodins respiratory model.

A further variant is the Francis model, which is more consistent with the Grodins model than MackeyGlass, but which also apparently incorrectly assumes $\mathrm{CO}_{2}$ at the brain is simply transported there from the lungs, ignoring the effect of metabolic production in the brain. Confusingly, it gives good agreement with experimental results.

It is possible to simplify the Grodins model to a single delay recruitment model ((4.7), with (4.11)), which can reasonably exhibit instability. Despite the dissimilarity with the derivation of the Mackey-Glass model, the instability criterion is effectively the same. The principal distinction is that the compartment volume parameter $K$ is made explicit in the Grodins model, whereas its meaning is unclear (and implicit) in the Mackey-Glass model.

In the reduced Grodins model, instability is caused by a fourfold increase in $\left(\frac{A \tau_{\mathrm{aB}} P^{*} G_{C}}{K_{B}}\right) \frac{Q_{B}^{*}}{Q^{*}}$, and this can be effected by a fourfold reduction in cardiac output (increasing the delay by four), or a fourfold increase in gain, if the fractional blood flow to the brain, $Q_{B}^{*} / Q^{*}$, remains the same; or a combination. This seems attractive, because the Francis et al. [13] experimental work indicates just such a fourfold increase in the critical parameter $\tau G_{C} P^{*}$. However, the buffering effect of the blood-brain barrier appears to inhibit instability, and this may explain the extreme artificial delays which Guyton et al. [15] had to use to induce instability in dogs.

In conclusion, it seems that models of Mackey-Glass-Grodins-Francis type may not quite have the power to deliver periodic breathing. As a final comment, we return to the issue of bicarbonate buffering, as described by (2.7). The forward reaction rate is

$$
R=k_{1}\left[\mathrm{CO}_{2}\right]-k_{-1}\left[\mathrm{HCO}_{3}^{-}\right]\left[\mathrm{H}^{+}\right]
$$

(the square brackets denote molar concentrations, $1 \mathrm{M}=1$ mole $^{-1}$ ), and is very fast, due to the presence of the enzyme carbonic anhydrase. According to Martin et al. [22], suitable values are

$$
k_{1}=2.73 \times 10^{4} \mathrm{~s}^{-1}, \quad k_{2}=3.437 \times 10^{10} \mathrm{M}^{-1} \mathrm{~s}^{-1},
$$

whence the equilibrium constant

$$
K_{1}=\frac{k_{1}}{k_{-1}} \approx 0.8 \times 10^{-6} \mathrm{M}
$$

With appropriate definition of units, terms in $R$ must be added to the compartment conservation laws (2.1). In [14], blood gas concentrations $C$ are measured as l(STPD) $\mathrm{l}^{-1}$, and these are converted to $\mathrm{M}$ by dividing by the molar volume $V_{M}=22.7 \mathrm{l}(\mathrm{STPD}) \mathrm{mole}^{-1}$; thus

$$
\left[\mathrm{CO}_{2}\right]=\frac{C}{V_{M}}
$$

To obtain (2.1) in terms of partial pressures, we used the approximate dissociation relation

$$
C=C_{0}+K_{\mathrm{CO}_{2}} P
$$


therefore, to include the reaction terms in (2.1) in units of $\mathrm{mm} \mathrm{Hg} \mathrm{s}^{-1}$, the equations are modified as follows:

$$
\begin{aligned}
& K_{\mathrm{L}}\left[\dot{P}_{\mathrm{aCO}_{2}}+\frac{V_{M} R_{a}}{K_{\mathrm{CO}_{2}}}\right]=-\dot{V} P_{\mathrm{aCO}_{2}}+863 K_{\mathrm{CO}_{2}} Q\left[P_{\mathrm{vCO}_{2}}-P_{\mathrm{aCO}_{2}}\right], \\
& K_{\mathrm{CO}_{2}} K_{\mathrm{B}}\left[\dot{P}_{\mathrm{BCO}_{2}}+\frac{V_{M} R_{B}}{K_{\mathrm{CO}_{2}}}\right]=M R_{\mathrm{BCO}_{2}}+K_{\mathrm{CO}_{2}} Q_{\mathrm{B}}\left[P_{\mathrm{aCO}_{2}}\left(t-\tau_{\mathrm{aB}}\right)-P_{\mathrm{BCO}_{2}}\right] \\
& -D_{\mathrm{CO}_{2}}\left[P_{\mathrm{BCO}_{2}}-P_{\mathrm{CSFCO}_{2}}\right] \text {, } \\
& K_{\mathrm{CO}_{2}} K_{\mathrm{T}}\left[\dot{P}_{\mathrm{TCO}_{2}}+\frac{V_{M} R_{T}}{K_{\mathrm{CO}_{2}}}\right]=M R_{\mathrm{TCO}_{2}}+\left(Q-Q_{B}\right) K_{\mathrm{CO}_{2}}\left[P_{\mathrm{aCO}_{2}}\left(t-\tau_{a T}\right)-P_{\mathrm{TCO}_{2}}\right] \text {, }
\end{aligned}
$$

where $R_{j}$ indicates the value of $R$ in compartment $j$.

The point here is similar to that made by Fowler et al. [12] in discussing an analogous problem of carbon chemistry in the ocean. The coefficients in the terms in $R$ in (6.6) are very large, so that each equation simply tells us that $R_{j} \approx 0$, i. e., the bicarbonate buffering system is in equilibrium. But this does not allow us to use (2.1)! Instead, it is necessary to include also the evolution of $\mathrm{HCO}_{3}^{-}$and $\mathrm{H}^{+}$as they travel round the system, and then deduce useful conservation laws corresponding to (2.1) by suitable elimination of the $R_{j}$ terms. This is a bigger enterprise, which we defer to future work; but it reinforces our conclusion that the Grodins model does not tell a whole, consistent story.

Acknowledgements. I congratulate Michael Mackey on reaching the age of 70, and for his achievements in the field of dynamic physiology, which he has largely invented. I also thank him for his comments on an earlier draft of this material. I acknowledge the support of the Mathematics Applications Consortium for Science and Industry (www.macsi.ul.ie) funded by the Science Foundation Ireland mathematics initiative grant 12/1A/1683.

\section{References}

[1] J. J. Batzel, H. T. Tran Modeling instability in the control system for human respiration: applications to infant nonREM sleep. Appl. Math. Comput. 110 (2000a), 1-51.

[2] J. J. Batzel, H. T. Tran Stability of the human respiratory control system. I. Analysis of a two-dimensional delay state-space model. J. Math. Biol. 41 (2000b), 45-79.

[3] J. J. Batzel, H. T. Tran Stability of the human respiratory control system. II. Analysis of a three-dimensional delay state-space model. J. Math. Biol. 41 (2000c), 80-102.

[4] A. Ben-tal, J. C. Smith Control of breathing: two types of delays studied in an integrated model of the respiratory system. Respir. Physiol. Neurobiol. 170 (2010), 103-112.

[5] D. W. Carley, D. C. Shannon A minimal mathematical model of human periodic breathing. J. Appl. Physiol. 65 (1988), 1400-1409.

[6] J.A. Cheyne A case of apoplexy in which the fleshy part of the heart was converted into fat. Dublin Hosp. Rep. 2 (1818), 216-219

[7] F. Dong, W. F. Langford Models of Cheyne-Stokes respiration with cardiovascular pathologies. J. Math. Biol. 57 (2008), $497-519$.

[8] A. ElHefnawy, G. M. Saidel, E. N. Bruce, N. S. Cherniack Stability analysis of $\mathrm{CO}_{2}$ control of ventilation. J. Appl. Physiol. 69 (1990), 498-503.

[9] A. C. Fowler, G. P. Kalamangalam, G. C. Kember A mathematical analysis of the Grodins model of respiratory control. IMA J. Math. Appl. Med. Biol. 10 (1993), 249-280.

[10] A. C. Fowler, G.P. Kalamangalam The role of the central chemoreceptor in causing periodic breathing. IMA J. Math. Appl. Med. Biol. 17 (2000), 147-167.

[11] A. C. Fowler, G. P. Kalamangalam Periodic breathing at high altitude. IMA J. Math. Appl. Med. Biol. 19 (2003), 293-313.

[12] A. C. Fowler, R.E. M. Rickaby, E. W. Wolff Exploration of a simple model for ice ages. Int. J. Geomath. 4 (2013), 227-297.

[13] D. P. Francis, K. Wilson, L. C. Davies, A. J.S. Coats, M. Piepoli Quantitative general theory for periodic breathing in chronic heart failure and its clinical implications. Circulation 102 (2000), 2,214-2,221.

[14] F.S. Grodins, J. Buell, A. J. Bart Mathematical analysis and digital simulation of the respiratory control system. J. Appl. Physiol. 22 (1967), 260-276. 
[15] A. C. Guyton, J. W. Crowell, J. W. Moore Basic oscillating mechanism of Cheyne-Stokes breathing. Amer. J. Physiol. 187 (1956), 395-398.

[16] A. C. Guyton, J. E. Hall Textbook of medical physiology, 11th ed. Elsevier, Pennsylvania, 2006.

[17] M. C. K. Khoo, R. E. Kronauer, K. P. Strohl, A. S. Slutsky Factors inducing periodic breathing in humans: a general model. J. Appl. Physiol. 53 (1982), 644-659.

[18] M. H. Kryger, T. Millar Cheyne-Stokes respiration: stability of interacting systems in heart failure. Chaos 1 (1991), 265-269.

[19] G.-S. Longobardo, B. Gothe, N. S. Cherniack Factors affecting respiratory system stability. Ann. Biomed. Engng. 17 (1989), 377-396.

[20] M. C. Mackey, L. Glass Oscillations and chaos in physiological control systems. Science 197 (1977), $287-289$.

[21] C. H. Manisty, K. Willson, R. Wensel, Z. I. Whinnett, J. E. Davies, W. L. G. Oldfield, J. Mayet, D. P. Francis Development of respiratory control instability in heart failure: a novel approach to dissect the pathophysiological mechanisms. J. Physiol. 577 (2006), 387-401.

[22] N. K. Martin, E. A. Gaffney, R. A. Gatenby, R. J. Gillies, I. F. Robey, P. K. Maini A mathematical model of tumour and blood pHe regulation: The $\mathrm{HCO}_{3}^{-} / \mathrm{CO}_{2}$ buffering system. Math. Biosci. 230 (2011), 1-11.

[23] J. D. Murray Mathematical biology. Springer-Verlag, Berlin, (2003).

[24] W. Stokes The disease of the heart and the aorta. Hodges and Smith, Dublin, Ireland, (1854).

[25] M. P. Ward, J. S. Milledge and J. B. West High altitude medicine and physiology, 3rd ed. Arnold, London, (2000).

[26] J. B. West Respiratory physiology - the essentials, 4th ed. Williams and Wilkins, Baltimore, (1990).

[27] J. B. West, R. M. Peters, G. K. H. Aksnes, J. S. Milledge, R. B. Schoene Nocturnal periodic breathing at 6300 and 8050 m. J. Appl. Physiol. 61 (1986), 280-287. 